\title{
Image Compression with Modified Skip line Encoding and Curve Fitting
}

\author{
Saumya Sadanandan \\ Dept of Computer Science and Engineering \\ National Institute of Technology, Calicut, INDIA
}

\author{
V. K. Govindan \\ Dept of Computer Science and Engineering \\ National Institute of Technology, Calicut, INDIA
}

\begin{abstract}
High quality digitized images have always been subject to high correlation: high image quality equals large file size. Image Compression is an important issue in Internet, mobile communication, digital library, digital photography, multimedia, teleconferencing and other applications. Application areas of Image Compression would focus on the problem of optimizing storage space and transmission bandwidth. Here a lossy method for image compression based on skip line encoding and curve fitting is proposed. Proposed approach involves two major processing steps: a lossless modified skip line encoding process to eliminate redundant scan lines in the image, and a lossy curve fitting based encoding for further redundancy elimination. The degree of compression is controlled based on the amount of loss that is affordable for applications making use of Peak Signal to Noise Ratio (PSNR) measure in the decision. The results obtained with the combined, modified skip line encoding and curve fitting approach, are analyzed in terms of compression ratio and PSNR. The approach provides improvements in compression ratio for all the tested images. The results obtained were found to be better than a state-of-the-art method in the literature.
\end{abstract}

\section{General Terms}

Image compression

\section{Keywords}

binary images, image compression, RLE encoding, skip-line encoding, curve fitting.

\section{INTRODUCTION}

Digital images require large number of bits to represent it and image compression is used to minimize the amount of memory required to represent an image. The objective of image compression is to reduce redundancy of the image data in order to store or transmit data in an efficient form. Image compression techniques are broadly classified into lossy compression and lossless compression. In lossless compression, the reconstructed image is same as the original image as there is no loss of information during compression and decompression process. In lossy compression, some loss of information is permitted without affecting the visual quality of the image. Benefits of image compression includes low storage space requirements, low communication bandwidth requirements, quicker sending and receiving of images, and less time on image viewing and loading. The objective of the proposed approach of compression is to store the data necessary to reconstruct a digital image using as little space as possible while maintaining enough visual detail demanded by the applications. Proposed algorithm is lossy, meaning that visual information is selectively discarded in order to improve the compression ratio. Generally, the neighbouring pixels in an image have high correlation to each other. Compression algorithms try to decorrelate the image pixels so that the resulting data require lower storage space for its representation.

\section{RELATED WORKS}

A good number of image compression methods has been proposed by different researchers based on techniques like dividing image into blocks $[1,2,3,4,5,6,7]$, compressions based on edge detection $[8,9]$, chain coding $[10,11]$, skip line encoding $[12,13]$ etc. These methods exploit the spatial redundancy or correlation between pixels in the image. There are also a number of compression techniques performed in frequency domain or other transformed domains [14]. Five major groups of works are: block truncation coding, compression techniques based on edge or block detection, chain coding, skipline encoding and techniques based on curve fitting. Some of the work belongs to these groups are briefly reviewed in the following:

\subsection{Block Truncation Coding}

The basic Block Truncation Coding (BTC) is proposed by Edward J. Delp [1]. It is a lossy fixed length compression that uses a $Q$ level quantization to quantize a local region of image. This method preserves the sample mean and standard deviation of a gray scale image. Two steps are there in the algorithm. In the first step, image is divided into non overlapping rectangular regions. Two luminance values are selected to represent each pixel in the block so that the sample mean and standard deviation of the reconstructed block are identical to those of the original block. With the help of a bit map for each block, decompression algorithm knows whether a pixel is brighter or darker than the average. BTC produces a fixed length binary representation of each block so that channel errors do not propagate in the decompressed image.

O. R. Mitchell et al. [2] proposed Multilevel Graphics Representation using block truncation coding. This method is suitable for the coding of images in which many gray levels are present. In multilevel graphics, a low pass (smoothing) operation followed by thresholding at the sample mean gives a bit plane. This will result in a reconstructed image of lowest mean square error. In this method mean and sample variance in each block is preserved.

A new version of region based BTC is proposed by J. Polec et al. [3]. It is a content related image compression technique. Here, they divided the image into quasi homogeneous regions sing a multi level thresholding algorithm and median filtering. Interiors of each segment is applied a region based truncation code related to $\mathrm{BTC}$.

C.K. Yang et al. [4] proposed Improving block truncation coding by line and edge information and adaptive bit plane selection for gray-scale image compression. A method using predefined line and edge bit planes to improve the BTC method is proposed. Corresponding to the visual continuity and discontinuity constraints, a set of line and edge bit plane is defined. The line and edge bit planes are defined independently of the image to be coded. The set of the entire 
best match predefined bit plane is classified and those types which occur more frequently in the set are picked out. Each block is finally coded by the values of the mean, the standard deviation and the index of the best match predefined bit plane [4]. Good compression performance with reasonable reconstructed image quality is reported.

Some of the recent works based on Block Truncation Coding incorporating fuzzy edges and Huffman codes are those proposed by T. M. Amarunnishad et al. [5, 6, 7]. In [5] and [6], BTC performance is enhanced employing fuzzy edge operator. In [7], BTC performance improvement is attempted making use of Huffman codes to compress bit planes numbers. In all the cases, substantial improvements in compression performances are reported.

\subsection{Compression Based on Edge or Block Detection}

A. Aggoun et al. [8] presented an image compression algorithm using local edge detection. In this, image is classified into visually active and visually continuous blocks which are then coded individually. It is based on a local histogram analysis. The differential image is obtained by applying a gradient operator. This image is divided into $4^{\times} 4$ non overlapping blocks and local histogram analysis is used to distinguish between visually continuous and visually active blocks. This algorithm works well for images with limited amount of texture and suitable for wireless image communications, where low power consumption is highly desirable.

U.Y. Desai et al. [9] proposed Edge and Mean based image compression algorithm for applications with very low bit rate. Spatial redundancy is reduced by extracting and encoding edge and mean information. They used Sobel operator for edge detection. A thinning operation is done to produce edges that are single pixel wide. This edge and mean based algorithm produces good quality images at very low bit rates. Sharp edges are very well preserved. Proposed method does not perform any texture coding, so there is some loss of texture.

\subsection{Chain Coding}

Lossless Chain Coder for Gray Edge Images is proposed by R. Redondo et al. [10]. This method provides lossless compression rates higher than the commonly used lossless methods. The method is based on searching pixels whose intensity is greater than zero. For such pixels, the amplitudes of their intensities and the required movements to reach them are stored. The image is scanned from left to right and from top to bottom until a non zero pixel is found. This pixel is called head of the chain. The searching process is performed clockwise and all stored pixels are marked for avoiding multiple coding. Huffman prefix codes and arithmetic codes are applied after the processing in order to obtain the final compressed code stream.

E. Tamir et al. proposed an efficient Chain Code encoding for segmentation based image compression [11]. In this, regions and lines are obtained as results of image segmentation, split and merge image compression or as the output of line and polygon algorithm. Lines and contours of uniform regions are encoded using chain code. The chain code is obtained in a way that is efficient with respect to bit rate and produces lossless contour and line encoding.

\subsection{Skip Line Encoding}

Skip-n-line encoding is proposed by A.A. Moinuddin, et al. [12] in 1997. If there is a high degree of correlation between successive scan lines, then there is no need to code each of them. Only one of them needs to be coded and the others may be skipped. While decoding, skipped lines are taken to be identical to the previous line. Lossless compression technique (Run length encoding) is used to compress the stored scan line. Skip one line encoding (S1LC) means if two successive scan lines are similar then code only one of them and skip the next line. That means in the best case only half of the total scan lines need to be coded.. Similarly, S2LC means code one line and skip next 2 lines. Here, in the best case only one third of the total scan lines need be run length coded. In 2010, Hao Sung et al. [13] proposed a Skip-line with threshold algorithm for Binary image compression.

\subsection{Compression Based on Curve Fitting}

In [15], Image compression using plane fitting with interblock prediction is proposed. Compression is achieved by plane fitting scheme. To reduce the computational requirements, the image is divided into non overlapping blocks and each block is considered as a 3D surface in which pixel gray value is the $\mathrm{z}$-axis and the block centre is the origin.

Chen et al. [16] proposed a Color Image Coding by using the technique of Surface Fitting in which digital RGB signals are converted to YIQ signals before coding. At the end of the receiver, the YIQ signals converted into RGB signals for the color monitor to display. Before the surface fitting procedure, high frequency parts of each component will be filtered out. The adaptive component coding procedure is performed on each component and is a three-step progressive approximation system which includes the initial surface fitting and two-pass improvements. The initial surface fitting process is performed to get the low level vision of the component. Two passes of adaptive improvement process are required to get satisfactory results.

Though there are a number of research works proposed by various researchers attempting to reduce the storage space and communication bandwidth, techniques for further improving the performances of data compression is always essential as the storage and communication requirements of present day devices increases day by day. Research leading to improved techniques providing even a small increase in compression ratio compared to the existing approaches is still important for saving storage space and bandwidth. In this paper, to achieve improved compression performance, a curve fitting routine is applied to the output of the modified skip line encoding preserving the visual content to the extend demanded by the applications.

\section{PROPOSED WORK}

A lossy image compression method based on global skip line encoding and piecewise polynomial curve fitting is proposed. Two major steps are involved in the proposed method. In the first step, global skip line encoding is applied to the image to eliminate the redundant scan lines in the image. In step 2, to the output obtained in step 1, a curve fitting algorithm (given in 3.2) is applied.

\subsection{Modified Skip Line Encoding}

Skip-n-line encoding is proposed by A.A. Moinuddin, et al. in 1997 [12]. If there is a high degree of correlation between successive scan lines, then there is no need to code each of them. Only one of them needs to be coded and the others may be skipped. While decoding, skipped lines are taken to be 
identical to the previous line. Lossless compression technique (Run length encoding) is used to compress the stored scan line.

In $[12,13]$, scan lines are skipped only if there is sufficient similarity between successive scan lines. In the proposed modified approach, scan line can be skipped if there is similar scan line anywhere in the image. In Figure 1 line 1 and line 2 are similar. So there is no need to store both the lines 1 and 2 . When comparing with line 3 , it is different from line 2 so store line 3 and we can skip line 4 . Next we compare line 4 and 5 and are different. In the modified approach, if we are about to store a new scan line we check whether a similar line is already stored or not. If it is already stored we update the index array which indicates the scan line to be substituted at the time of decoding.

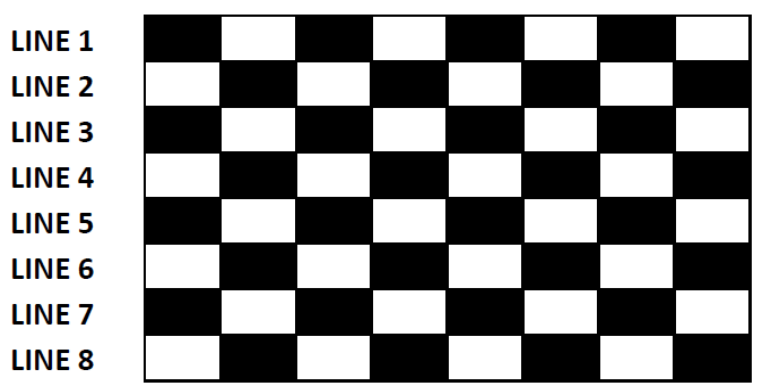

Figure 1. Image before compression

Figure 2 shows compressed form of image in Figure 1. It has only two lines. Additionally there is an index storing array which is used for decompression.

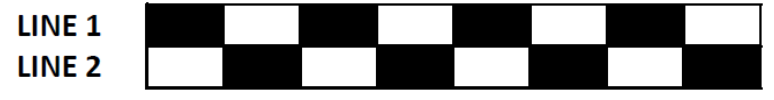

Figure 2. Image after compression

\subsection{Curve Fitting}

Splines are piecewise polynomials with pieces that are smoothly connected together. The joining points of the polynomials are called knots. For a spline of degree n, each segment is a polynomial of degree $n$, which would suggest that we need $n+1$ coefficient to describe each piece. A piecewise polynomial function $\mathrm{f}(\mathrm{x})$ is obtained by dividing of $X$ into contiguous intervals, and representing $f(x)$ by a separate polynomial in each interval. A simple cubic spline function can be applied in the form of the following equation [17]:

$$
S=a+b x+c x^{2}+d x^{3}
$$

The parameters $a, b, c$ and $d$ are obtained by the method of least-square-error approximation from the solution of the following system of equations [17]:

$$
\left[\begin{array}{cccc}
n & \sum_{i=1}^{n} x_{i} & \sum_{i=1}^{n} x_{i}^{2} & \sum_{i=1}^{n} x_{i}^{3} \\
\sum_{i=1}^{n} x_{i} & \sum_{i=1}^{n} x_{i}^{2} & \sum_{i=1}^{n} x_{i}^{3} & \sum_{i=1}^{n} x_{i}^{4} \\
\sum_{i=1}^{n} x_{i}^{2} & \sum_{i=1}^{n} x_{i}^{3} & \sum_{i=1}^{n} x_{i}^{4} & \sum_{i=1}^{n} x_{i}^{5} \\
\sum_{i=1}^{n} x_{i}^{3} & \sum_{i=1}^{n} x_{i}^{4} & \sum_{i=1}^{n} x_{i}^{5} & \sum_{i=1}^{n} x_{i}^{6}
\end{array}\right] *\left[\begin{array}{l}
a \\
b \\
c \\
d
\end{array}\right]=\left[\begin{array}{l}
\sum_{i=1}^{n} y_{i} \\
\sum_{i=1}^{n} x_{i} y_{i} \\
\sum_{i=1}^{n} x_{i}^{2} y_{i} \\
\sum_{i=1}^{n} x_{i}^{3} y_{i}
\end{array}\right]
$$

Where $n$ is the number of pairs of data values in the interval $[a, b]$.
In the case of the non uniform variation of data that occurs in most engineering problems, a single spline curve cannot be applied. Instead, it is necessary to use piecewise spline curves to approximate such data. Therefore, given a set of points with associated function values and first derivatives, we can determine a sequence of polynomials that interpolate the data. Let $y=a x+b$ be a first order polynomial equation. Using a linear polynomial, the Least Square Curve Fitting is concerned with approximating the data points using parameters $a$ and $b$ such that the sum of the squared error is minimum. A set of data are given that are approximated using these parameters. Reconstructed data points give approximated values using curve fitting. If the curve fitting is accurate it means that calculated values and the original values are same. The main objective of this work is to find efficient representations of an image using polynomial fitting. To find the model parameters, the Mean Square Error (MSE) minimization method is used. The first order polynomial is of special interest due to its computational simplicity. To obtain a better quality, one has to increase polynomial order or decrease the block size and pay the price by decreasing compression ratio. Increasing the polynomial order improves the quality of the resultant image. The price paid is the reduction in compression ratio. The higher order polynomial schemes have higher computational burden and inferior performance compared to that of the plane fitting scheme. The output obtained after applying modified skip line encoding is a matrix of pixel intensities with redundant lines eliminated. This matrix is divided into sub blocks of variable sizes based on a specified PSNR value. Least Square Curve Fitting is concerned with calculating the data using parameters ' $a_{0}$ ', ' $a_{1}$ ' in first order curve fitting. Reconstructed data points give approximated values using curve fitting.

We store only few parameters for a number of pixels using a least square algorithm.

$$
\text { Minimize } \sum_{x}(g(x, y)-f(x, y))^{2}
$$

Where $g(x, y)$ is the original image and $f(x, y)=a_{0}+a_{1} x+a_{2} y$. Parameter values $\mathrm{a}_{0}, \mathrm{a}_{1}, \mathrm{a}_{2}$ are stored for all blocks.

\section{Algorithm}

Input: An image with all redundant scan lines eliminated by applying modified skip line encoding.

\section{Repeat for each row}

1. Take a new unprocessed block of size of 2 .

2. If the block size exceeds total available size, then set block size as maximum available power of 2 . Do steps 3,4 , and 6 .

3. Fit a first order polynomial to the block.

4. Reconstruct the block

5. If the PSNR of the reconstructed block is greater than specified PSNR then

5.1 Double the block size 5.2 Go to step 2

6. Else

6.1 Store the values of previous iteration (If it is first iteration, store the same value) and number of elements in that block. 
6.2 Go to step 1 if some more data is left in that row.

\section{Output:}

1. A 'coefficient matrix', the matrix storing coefficients obtained for each block with curve fitting.

2. A 'no_of_elements_per_block', the matrix containing number of pixel intensities in each block.

\section{EXPERIMENTAL RESULTS}

Results obtained with the proposed approach are analyzed in terms of compression ratio (CR) and Peak Signal to Noise Ratio (PSNR).

\subsection{Compression Ratio (CR)}

The percent compression ratio defined as given below is used to evaluate the performance of the approach:

$\%$ Compression ratio $=[($ original size - compressed size)/ original size] $* 100$.

\subsection{Peak Signal to Noise Ratio (PSNR)}

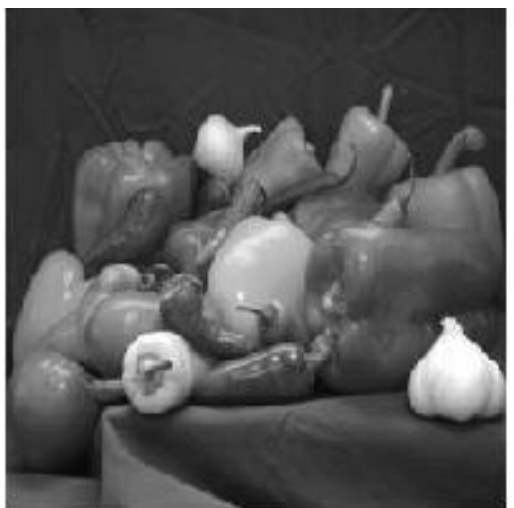

(a)

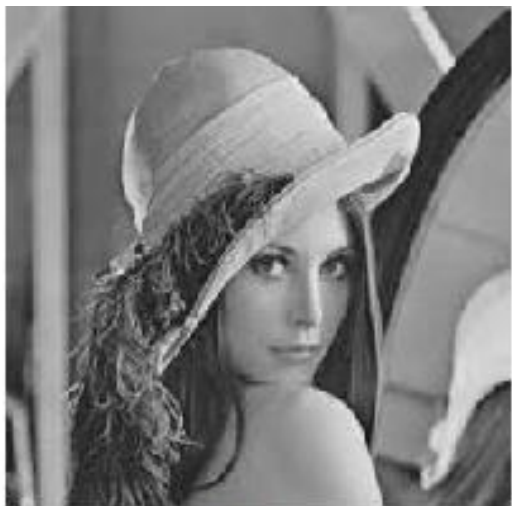

(b)
Peak signal to noise ratio (PSNR) is defined as

$$
P S N R=20 \log _{10} \frac{255}{\sqrt{M S E}}
$$

Where

$$
\text { MSE }=\frac{1}{M * N} \sum_{i=1}^{M} \sum_{j=1}^{N}(g(i, j)-f(i, j))^{2}
$$

Where $f(i, j)$ is the reconstructed image and $g(i, j)$ is the original image, and $\mathrm{M}, \mathrm{N}$ are the dimensions of the image.

\subsection{Performance of modified skip line encoding with curve fitting}

When modified skip line encoding is combined with curve fitting, the encoding techniques become a lossy one. The sample images used for testing are given in Fig.3. The result obtained with this combined approach is found to be better than [13] in terms of compression ratio and PSNR value. The results obtained with both the methods are given in Table 1. Figures 4 to 6 provide the graphical representation of comparative compression performances of the proposed approach and the skip line encoding technique given in [13] for Pepper, Lena and Cameraman images. The graphs demonstrate the superior performances of the proposed approach in all the three cases.

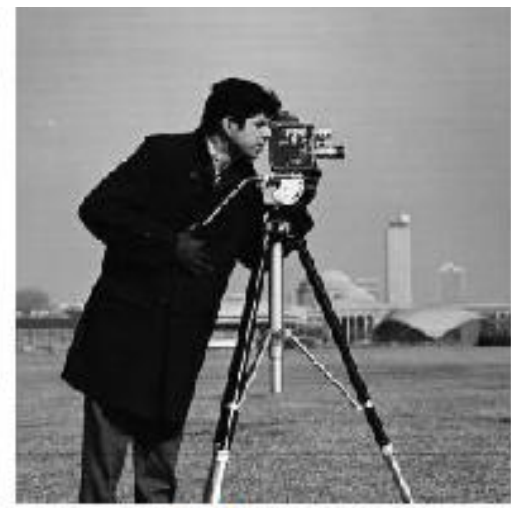

(c)

Figure 3. Images used for testing a) Pepper b) Lena c) Cameraman 
Table 1. Results of proposed scheme and lossy skip line encoding

\begin{tabular}{|c|c|c|c|c|}
\hline \multirow{3}{*}{ IMAGE } & \multicolumn{2}{|c|}{ LOSSY SKIP LINE ENCODING [13] } & \multicolumn{2}{c|}{ PROPOSED METHOD } \\
\cline { 2 - 5 } & CR & PSNR(db) & CR & PSNR(db) \\
\hline \multirow{3}{*}{ PEPPER } & 46.38 & 38 & 86.61 & 38 \\
\cline { 2 - 5 } & 76.44 & 31 & 92.65 & 27.5 \\
\cline { 2 - 5 } & 84.92 & 27.5 & 57.54 & 41 \\
\hline \multirow{3}{*}{ LENA } & 30.87 & 41.29 & 91.11 & 26 \\
\cline { 2 - 5 } & 71.86 & 30.22 & 95.46 & 36.84 \\
\cline { 2 - 5 } & 82.73 & 26.76 & 57.86 & 33.62 \\
\hline \multirow{3}{*}{ CAMERAMAN } & 38.92 & 37 & 85.78 & 31 \\
\cline { 2 - 5 } & 43.11 & 34 & 89.20 & 27.49 \\
\hline
\end{tabular}

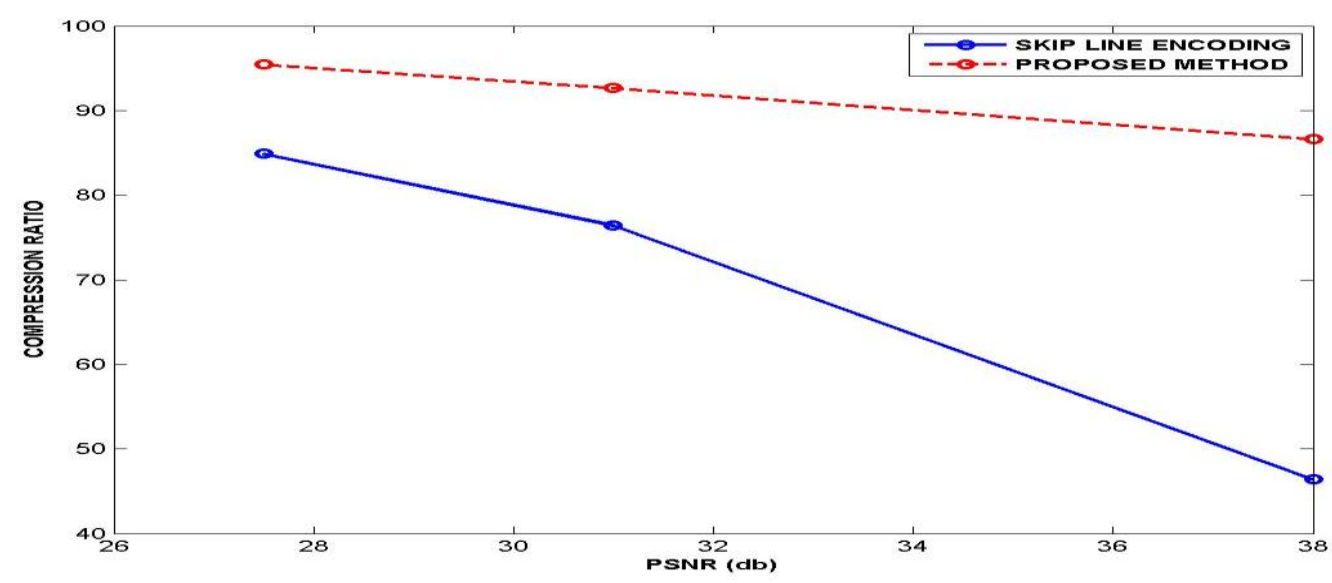

Figure. 4 Comparison of proposed scheme with skip line encoding for pepper image

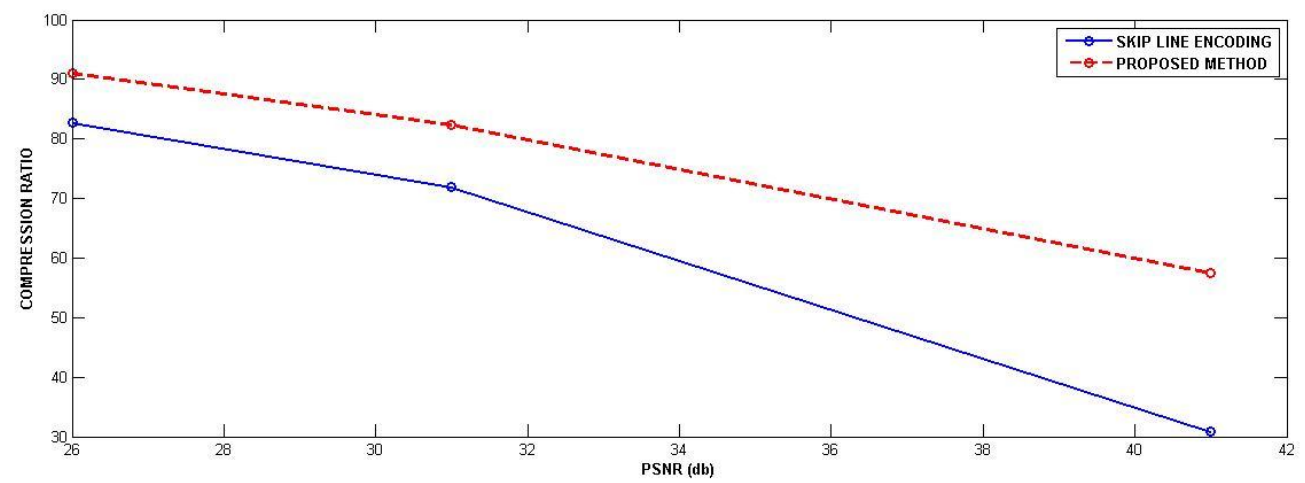

Figure. 5 comparison of proposed scheme with skip line encoding for lena image 


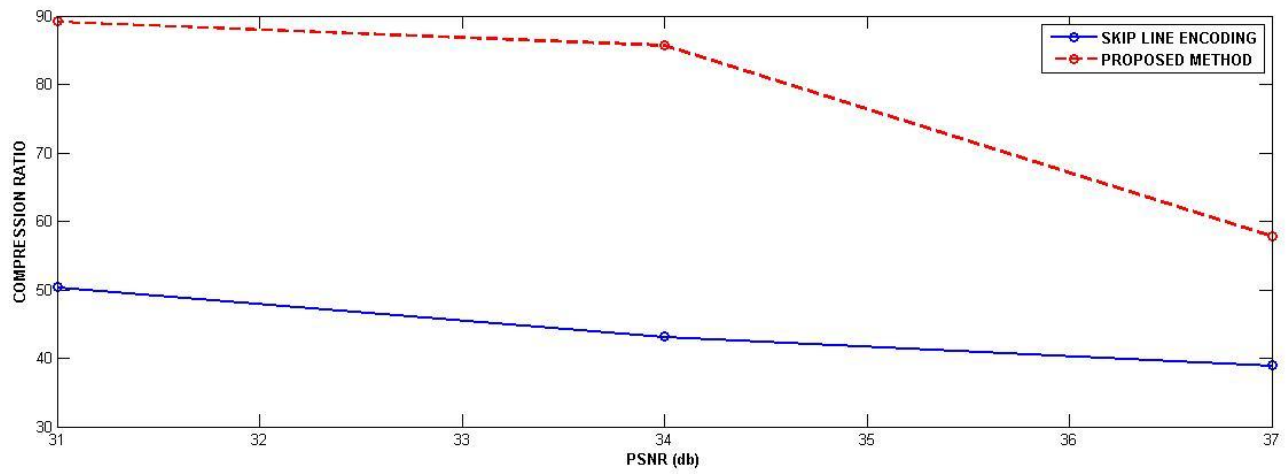

Figure.6 Comparison of proposed scheme with skip line encoding for cameraman image

\section{CONCLUSION}

Though the storage device price decreases and size increases, the storage requirements are still increasing as the present day images/ data are of huge size. Research leading to improved techniques providing even a small increase in compression ratio compared to the existing approaches is still important for saving storage space and bandwidth. To improve the compression ratio a curve fitting routine is applied to the output obtained in the modified skip line encoding preserving the visual content demanded by the application. During the compression process some information is lost due to approximate fitting of the curve on pixel data. This loss of information results in degradation in subjective quality of an image and increases the Mean Square Error. This made the

\section{REFERENCE}

[1] E.J. Delp, M. Saenz and Salma, article BLOCK TRUNCATION CODING (BTC), 2010.

[2] O.R Mitchell and E.J. Delp, "Multilevel graphics representation using block truncation coding", proceedings of the IEEE, vol.68, no.7, pp.868-873,July 1980.

[3] J. Polec and J. Pavlovicova,; , "A new version of region based BTC," EUROCON'2001, Trends in communications, International Conference on. , vol.1, no., pp.88-90 vol.1, 4-7 July 2001.

[4] C.K. Yang, and W.H. Tsai, Improving block truncation coding by line and edge information and adaptive bit plane selection for gray-scale image compression, Pattern recognition letters, volume.16,number1,pages $=67$ 75,1995 .

[5] T.M. Amarunnishad, V. K. Govindan and Abraham T. Mathew, Improved BTC image compression using a fuzzy complement edge operator, signal Processing, vol88, issue 12, (2008)2989-2997, Elsevier 2008.

[6] T.M. Amarunnishad, V. K. Govindan and Abraham T. Mathew, Use of Fuzzy Edge Image in Block Truncation Coding for Image compression, International Journal of signal Processing, Vol 4, N0 3, pp 215-221, 2008.

[7] T.M. Amarunnishad, V. K. Govindan and Abraham T. Mathew, Block Truncation Coding with Huffman coding, Journal of medical imaging and health informatics, Vol. 1, No. 2, pp170-176, 2011. algorithm a lossy compression technique. The degree of compression can be adjusted, allowing a selectable tradeoff between storage size and image quality. When we increase the block size, the Compression Ratio also increases but the quality of the image decreases. Decreasing block size gives better quality of the image as compared to bigger block size but it gives lower compression ratio. Curve order also affects the compression ratio and quality of an image. When we increase the curve order, image quality increases but $\mathrm{CR}$ decreases.

[8] A. Aggoun and A. El-Mabrouk; , "Image compression algorithm using local edge detection," Wireless Image/Video Communications, 1996., First International Workshop on , vol., no., pp.68-73, 4-5 Sep 1996.

[9] U.Y. Desai, M. M. Mizuki and I. Masakiand Horn; B.K.P., Edge and mean based image compression, 1996.

[10] R. Redondo and G. Cristobal; "Lossless chain coder for gray edge images," Image Processing, 2003. ICIP 2003. Proceedings. 2003 International Conference on , vol.2, no., pp. II- 201-4 vol.3, 14-17 Sept. 2003

[11] D. E. Tamir , K. Phillip and Abdul-Karim, , "Efficient chain-code encoding for segmentation-based image compression," Data Compression Conference, 1996. DCC '96. Proceedings, vol., no., pp.455, Mar/Apr 1996.

[12] Moinuddin A.A, E. Khan, and F. Ghani. An effficient technique for storage of two-tone images. Consumer Electronics, IEEE Transactions on, 43(4):1312-1319, 1997.

[13] H. Sung and W.Y. Kuo. A skip-line with threshold algorithm for binary image compression. In Image and Signal Processing (CISP), $2010 \quad 3^{\text {rd }}$ International Congress on, volume 2, pages 515-523. IEEE, 2010.

[14] M.B.Akhtar, A.M. Qureshi and Qamar-ul-Islam, "Optimized run length coding for jpeg image compression used in space research program of IST," Computer Networks and Information Technology (ICCNIT), 2011 International Conference on , vol., no., pp.81-85, 11-13 July 2011. 
[15] Ameer, Salah, and Otman Basir. "Image compression using plane fitting with inter block prediction." Image and Vision Computing 27.4 (2009): 385-390.

[16] Chen, Y. S., H. T. Yen, and W. H. Hsu. "Color image coding by using the technique of surface fitting." Pattern Recognition, 1992. Vol. III. Conference C: Image, Speech and Signal Analysis, Proceedings., 11th IAPR International Conference on. IEEE, 1992
[17] Ichida, K., F. Yoshimoto, and T. Kiyono. "Curve fitting by a piecewise cubic polynomial." Computing 16.4 (1976): 329-338.

[18] Zamani, Mehdi. "A simple piecewise cubic spline method for approximation of highly nonlinear data." Advances in Molecular Imaging 4 (2012). 